\title{
ON HYBRID SEQUENCES BUILT FROM NIEDERREITER-HALTON SEQUENCES AND KRONECKER SEQUENCES
}

\author{
ROSWITHA HOFER and PETER KRITZER ${ }^{凶}$
}

(Received 10 November 2010)

\begin{abstract}
We discuss the distribution properties of hybrid sequences whose components stem from NiederreiterHalton sequences on the one hand, and Kronecker sequences on the other. In this paper, we give necessary and sufficient conditions on the uniform distribution of such sequences, and derive a result regarding their discrepancy. We conclude with a short summary and a discussion of topics for future research.
\end{abstract}

2010 Mathematics subject classification: primary 11K06, 11K38; secondary 11L07.

Keywords and phrases: uniform distribution modulo one, discrepancy, Kronecker sequence, NiederreiterHalton sequence.

\section{Introduction}

In many applications, for example financial mathematics, one is interested in numerically approximating the value of the integral of a function $f:[0,1]^{s} \rightarrow \mathbb{R}$,

$$
I_{s}(f):=\int_{[0,1]^{s}} f(\boldsymbol{x}) d \boldsymbol{x} .
$$

One way of accomplishing this task is to use a quasi-Monte Carlo (QMC) rule by which the integral is approximated,

$$
Q_{N, s}(f):=\frac{1}{N} \sum_{n=0}^{N-1} f\left(\boldsymbol{x}_{n}\right),
$$

where $\left(\boldsymbol{x}_{n}\right)_{n=0}^{N-1}$ is a finite sequence (or the portion of the first $N$ points of an infinite sequence) of deterministically chosen points in $[0,1)^{s}$. It is well known in the theory of QMC methods that point sets which are evenly distributed in the unit cube yield a low integration error when a QMC algorithm is used (see, among many others, $[1,3,11,13,16])$. Whenever we speak of a point set in the following, we mean a finite or infinite sequence of points, where points may occur repeatedly.

The authors gratefully acknowledge the support of the Austrian Science Fund (Project P21943).

(C) 2011 Australian Mathematical Publishing Association Inc. 0004-9727/2011 \$16.00 
Due to the use of uniformly distributed point sets in numerical integration, the search for point sets which can be effectively constructed and used in QMC integration rules has been a very active area of mathematical research in recent decades. As we stated above, the underlying problem is to find point sets with good distribution properties. Let us, therefore, first give the formal definition of uniform distribution modulo one.

DEFINITION 1.1. Let $S=\left(z_{n}\right)_{n \geq 0}$ be a sequence of points in $[0,1)^{s}$. We say that $S$ is uniformly distributed modulo one if, for all subintervals $J$ of $[0,1)^{s}$, it is true that

$$
\lim _{N \rightarrow \infty} \frac{\#\left\{0 \leq n<N: z_{n} \in J\right\}}{N}=\lambda(J),
$$

where $\lambda(J)$ denotes the Lebesgue measure of $J$.

One way of finding out whether a given sequence of points is uniformly distributed modulo one or not is to use Weyl's criterion, which we make use of in this paper as well. For a proof, see, for example, $[1,11]$.

THEOREM 1.2 (Weyl's criterion). A sequence $S=\left(z_{n}\right)_{n \geq 0}$ of points in $[0,1)^{s}$ is uniformly distributed modulo one if and only if

$$
\lim _{N \rightarrow \infty} \frac{1}{N} \sum_{n=0}^{N-1} e^{2 \pi \mathrm{i} \boldsymbol{h} \cdot z_{n}}=0
$$

for all nonzero vectors $\boldsymbol{h} \in \mathbb{Z}^{s}$.

A very prominent example of a class of uniformly distributed sequences is provided by the class of Kronecker sequences, which are defined as follows.

Definition 1.3. Let $s \in \mathbb{N}$ and $\boldsymbol{\alpha}=\left(\alpha_{1}, \ldots, \alpha_{s}\right) \in[0,1)^{s}$. Then the sequence $\left(\boldsymbol{y}_{n}\right)_{n \geq 0}$, where $\boldsymbol{y}_{n}:=\left(\left\{n \alpha_{1}\right\}, \ldots,\left\{n \alpha_{s}\right\}\right)$, in the sequel often denoted by $(\{n \boldsymbol{\alpha}\})_{n \geq 0}$, is called a Kronecker sequence. Here $\{\cdot\}$ gives the fractional part of a real number.

It is a well-known fact (see again $[1,3,11,13,16]$ ) that a Kronecker sequence is uniformly distributed if and only if $1, \alpha_{1}, \ldots, \alpha_{s}$ are linearly independent over $\mathbb{Q}$.

Another very important class of uniformly distributed sequences are digital $(t, s)$ sequences over $\mathbb{Z}_{q}$ as introduced by Niederreiter (see $[15,16]$ ) or, more generally, digital (T, $s$ )-sequences over $\mathbb{Z}_{q}$ as introduced by Larcher and Niederreiter [12]. In this case, $\mathbf{T}: \mathbb{N} \rightarrow \mathbb{N}_{0}$ is a quality function for the uniformity of the sequence. The smaller the values of $\mathbf{T}$ are, the better the distribution properties of the sequence. The precise definition of a digital $(\mathbf{T}, s)$-sequence is as follows. For the sake of simplicity we do not distinguish between the set of residue classes modulo $q$ and the set of integers $\{0,1, \ldots, q-1\}$ here and later on.

Definition 1.4. Let $s$ be a dimension and let $q$ be a prime. Let $C_{1}, \ldots, C_{s}$ be $\mathbb{N} \times \mathbb{N}_{0}$ matrices over the finite field $\mathbb{Z}_{q}$. We construct a sequence $\left(\boldsymbol{x}_{n}\right)_{n \geq 0}$, $\boldsymbol{x}_{n}=\left(x_{n}^{(1)}, \ldots, x_{n}^{(s)}\right), n \in \mathbb{N}_{0}$, by generating the $j$ th coordinate of the $n$th point, $x_{n}^{(j)}$, 
as follows. Represent $n=n_{0}+n_{1} q+n_{2} q^{2}+\cdots$ in base $q$. Then we set

$$
C_{j} \cdot\left(n_{0}, n_{1}, \ldots\right)^{\top}=:\left(y_{1}^{(j)}, y_{2}^{(j)}, \ldots\right)^{\top} \in \mathbb{Z}_{q}^{\mathbb{N}}
$$

and

$$
x_{n}^{(j)}:=\frac{y_{1}^{(j)}}{q}+\frac{y_{2}^{(j)}}{q^{2}}+\cdots .
$$

For every $m \in \mathbb{N}$ let $\mathbf{T}(m)$, satisfying $0 \leq \mathbf{T}(m) \leq m$, be such that for all $d_{1}, d_{2}, \ldots, d_{s} \in \mathbb{N}_{0}$, with $d_{1}+\cdots+d_{s}=m-\mathbf{T}(m)$, the $(m-\mathbf{T}(m)) \times m$ matrix consisting of the

left upper $d_{1} \times m$-submatrix of $C_{1}$ together with the

left upper $d_{2} \times m$-submatrix of $C_{2}$ together with the

$$
\text { left upper } d_{s} \times m \text {-submatrix of } C_{s}
$$

has rank $m-\mathbf{T}(m)$. Then $\left(\boldsymbol{x}_{n}\right)_{n \geq 0}$ is called a digital $(\mathbf{T}, s)$-sequence over $\mathbb{Z}_{q}$. If $\mathbf{T}$ is minimal with this property, we speak of a strict digital $(\mathbf{T}, s)$-sequence. If $\mathbf{T}(m) \leq t$ for all $m$, then we speak of a digital $(t, s)$-sequence.

The traditional approach in using QMC methods is to use the first $N$ points of uniformly distributed sequences such as the ones outlined above for the numerical integration algorithm. Modifying this approach, Spanier proposed mixing quasiMonte Carlo and Monte Carlo methods in [20]. To be more precise, Spanier proposed considering sequences of points where $s$-dimensional vectors of QMC point sets and $d$-dimensional (pseudo-)random vectors are concatenated, that is, one obtains an $(s+d)$-dimensional 'mixed' or 'hybrid' sequence.

In this paper, we do not consider the mixture of (pseudo-)random and QMC point sets, but study a slightly modified concept of a hybrid sequence, namely one that is obtained by mixing different QMC point sets. Thus, whenever we speak of a hybrid sequence in the sequel, we refer to a sequence that is obtained by concatenating the components of two or more quasi-Monte Carlo point sets. One way of doing so is to consider sequences which are a mixture of digital $(t, s)$-sequences in different bases. This type of sequence was studied for the first time in [7]; Hofer and Larcher showed further results in [5, 6, 9] and introduced the name Niederreiter-Halton sequences, which we make use of as well.

Definition 1.5. Let $v, w_{1}, \ldots, w_{v}$ be positive integers and let $q_{1}, \ldots, q_{v}$ be different primes. For $l \in\{1, \ldots, v\}$ and $j \in\left\{1, \ldots, w_{l}\right\}$, let $C^{(l, j)}$ be $\mathbb{N} \times \mathbb{N}_{0}$ matrices over $\mathbb{Z}_{q_{l}}$, of the form

$$
C^{(l, j)}:=\left(\begin{array}{cccc}
\gamma_{1,0}^{(l, j)} & \gamma_{1,1}^{(l, j)} & \gamma_{1,2}^{(l, j)} & \ldots \\
\gamma_{2,0}^{(l, j)} & \gamma_{2,1}^{(l, j)} & \gamma_{2,2}^{(l, j)} & \ldots \\
\gamma_{3,0}^{(l, j)} & \gamma_{3,1}^{(l, j)} & \gamma_{3,2}^{(l, j)} & \ldots \\
\vdots & \vdots & \vdots & \ddots
\end{array}\right) \in \mathbb{Z}_{q_{l}}^{\mathbb{N} \times \mathbb{N}_{0}}
$$


We denote row $r$ of matrix $C^{(l, j)}$ by $\gamma_{r}^{(l, j)}=\left(\gamma_{r, k}^{(l, j)}\right)_{k \geq 0}$ in $\mathbb{Z}_{q_{l}}^{\mathbb{N}_{0}}$. We define a sequence $\left(\boldsymbol{x}_{n}\right)_{n \geq 0}$ in $[0,1)^{s}, s:=w_{1}+\cdots+w_{v}$, by

$$
\boldsymbol{x}_{n}:=\left(x_{n}^{(1,1)}, \ldots, x_{n}^{\left(1, w_{1}\right)}, \ldots, x_{n}^{(v, 1)}, \ldots, x_{n}^{\left(v, w_{v}\right)}\right) .
$$

The component $x_{n}^{(l, j)}$, for $j \in\left\{1, \ldots, w_{l}\right\}, l \in\{1, \ldots, v\}$, is generated as follows. Let $n=n_{0}^{(l)}+n_{1}^{(l)} q_{l}+n_{2}^{(l)} q_{l}^{2}+\cdots$ be the base $q_{l}$ representation of $n$ for $l \in\{1, \ldots, v\}$. Then we set

$$
C^{(l, j)} \cdot\left(n_{0}^{(l)}, n_{1}^{(l)}, \ldots\right)^{\top}=:\left(y_{1}^{(l, j)}, y_{2}^{(l, j)}, \ldots\right)
$$

and

$$
x_{n}^{(l, j)}:=\frac{y_{1}^{(l, j)}}{q_{l}}+\frac{y_{2}^{(l, j)}}{q_{l}^{2}}+\cdots .
$$

Note that, in Definition $1.5, y_{r}^{(l, j)}$ can be interpreted as a weighted sum of digits $s_{q_{l}, \gamma_{r}^{(l, j)}}(n)$ taken modulo $q_{l}$, where

$$
s_{q_{l}, \gamma_{r}^{(l, j)}}(n):=n_{0}^{(l)} \gamma_{r, 0}^{(l, j)}+n_{1}^{(l)} \gamma_{r, 1}^{(l, j)}+\cdots
$$

For the sake of simplicity we restrict ourselves to generating matrices $C^{(l, j)}$ of finite column lengths, that is, for each choice of $l, j$, and $k, \gamma_{r, k}^{(l, j)}=0$ for all sufficiently large $r \in \mathbb{N}$.

As can be seen from the definition, a Niederreiter-Halton sequence is built by juxtaposing $v w_{l}$-dimensional digital $\left(\mathbf{T}, w_{l}\right)$-sequences in base $q_{l}$ for pairwise coprime bases. In [6] all uniformly distributed Niederreiter-Halton sequences were classified and the astonishing fact was proved that the obviously necessary condition that each of the $v$ digital component sequences is uniformly distributed is also sufficient.

In this paper, we wish to take up and further advance an idea due to Niederreiter $[17,18]$, who studied the distribution properties of sequences obtained by mixing Halton sequences and Kronecker sequences (see also [8]). The subject of the paper is hybrid sequences obtained by mixing Niederreiter-Halton sequences (which are a generalization of Halton sequences; see, for example [7]) and Kronecker sequences.

The rest of the paper is structured as follows. In Section 2 we discuss the circumstances under which a hybrid sequence made up of a Niederreiter-Halton sequence and a Kronecker sequence is uniformly distributed. Indeed, we show necessary and sufficient conditions for uniform distribution. In Section 3 we add a quantitative result as we show a theorem on the discrepancy of our hybrid sequences. The discrepancy of a point set, the precise definition of which will be stated in Section 3, is a way of measuring its quality of distribution. Finally, we conclude in Section 4 and discuss open problems for future research.

\section{Results on uniform distribution}

In this section we derive results on the uniformity of distribution of hybrid sequences which are obtained by mixing Niederreiter-Halton and Kronecker sequences. 
One of the main results in this paper is the following theorem, which states that whenever we mix a uniformly distributed Niederreiter-Halton sequence with a uniformly distributed Kronecker sequence we obtain another uniformly distributed sequence.

THEOREM 2.1. Let $\left(\boldsymbol{x}_{n}\right)_{n \geq 0}$ be an s-dimensional Niederreiter-Halton sequence as in Definition 1.5 and $\left(\boldsymbol{y}_{n}\right)_{n \geq 0}$ be a d-dimensional Kronecker sequence as in Definition 1.3. Then the $(s+d)$-dimensional sequence $\left(z_{n}\right)_{n \geq 0}$ with $z_{n}:=\left(\boldsymbol{x}_{n}, \boldsymbol{y}_{n}\right)$, $n \geq 0$, is uniformly distributed modulo one if and only if $\left(\boldsymbol{x}_{n}\right)_{n \geq 0}$ and $\left(\boldsymbol{y}_{n}\right)_{n \geq 0}$ are both uniformly distributed modulo one.

The proof will mainly be based on a proposition about exponential sums and $q$-additive functions. We call a function $g: \mathbb{N}_{0} \rightarrow \mathbb{R} q$-additive if $g(0)=0$ and

$$
g\left(n_{0}+n_{1} q+n_{2} q^{2}+\cdots\right)=g\left(n_{0}\right)+g\left(n_{1} q\right)+g\left(n_{2} q^{2}\right)+\cdots \quad \forall n \in \mathbb{N}_{0},
$$

where $n_{0}+n_{1} q+n_{2} q^{2}+\cdots$ is the unique base $q$ representation of $n$. Furthermore, we make use of the following notation. For a real number $x$, we define $\|x\|$ to be the distance to the nearest integer, that is, $\|x\|$ is the minimum of $\{x\}$ and $1-\{x\}$, with $\{\cdot\}$ denoting the fractional part of a real number.

Proposition 2.2. Let $d \in \mathbb{N}$ and $q_{1}, \ldots, q_{d} \geq 2$ be pairwise coprime integers. For each $i \in\{1, \ldots, d\}$, let $g^{(i)}: \mathbb{N}_{0} \rightarrow \mathbb{R}$ be a $q_{i}$-additive function. Then

$$
\lim _{N \rightarrow \infty} \frac{1}{N} \sum_{n=0}^{N-1} \prod_{i=1}^{d} e^{2 \pi \mathrm{i} g^{(i)}(n)}=0
$$

if, for at least one $i \in\{1, \ldots, d\}$,

$$
\sum_{k=0}^{\infty} \tau_{k}^{(i)}=\infty
$$

where $\tau_{k}^{(i)}$ is defined as follows. We write, for $a \in\{0, \ldots, q-1\}$ and $k \geq 0$,

$$
\begin{aligned}
\theta_{k}^{(i)}(a) & :=g^{(i)}\left((a+1) q_{i}^{k}\right)-g^{(i)}\left(a q_{i}^{k}\right)-g^{(i)}\left(q_{i}^{k}\right), \\
\delta_{k}^{(i)} & :=\max \left\{4\left\|\theta_{k}^{(i)}(a)\right\|^{2}: 1 \leq a \leq q_{i}-2\right\},
\end{aligned}
$$

and we set $\delta_{k}^{(i)}:=0$ if $q_{i}=2$. Now we define

$$
\tau_{k}^{(i)}:= \begin{cases}\max \left\{\delta_{k}^{(i)}, \delta_{k+1}^{(i)}\right\} / q_{i}^{2} & \text { if this expression is different from } 0 \\ \left\|g^{(i)}\left(q_{i}^{k+1}\right)-q_{i} g^{(i)}\left(q_{i}^{k}\right)\right\|^{2} / 4 & \text { otherwise. }\end{cases}
$$

PROOF. See [10, Proposition 1].

Proof of TheOrem 2.1. Necessity of uniform distribution of the component sequences $\left(\boldsymbol{x}_{n}\right)_{n \geq 0}$ and $\left(\boldsymbol{y}_{n}\right)_{n \geq 0}$ is obvious. For the proof of sufficiency we show that each interval of a certain form contains the correct number of points, that is, the number 
of points is proportional to the volume of the interval. Let

$$
J:=J_{s} \times J_{d}
$$

with

$$
J_{s}:=\prod_{l=1}^{v} \prod_{j=1}^{w_{l}}\left[\frac{a^{(l, j)}}{q_{l}^{d^{(l, j)}}}, \frac{a^{(l, j)}+1}{q_{l}^{d^{(l, j)}}}\right), \quad J_{d}:=[\mathbf{0}, \boldsymbol{\eta}),
$$

where the nonnegative integers $d^{(l, j)}$ and $a^{(l, j)}<q_{l}^{d^{(l, j)}}$ are fixed for all $l \in$ $\{1, \ldots, v\}, j \in\left\{1, \ldots, w_{l}\right\}$ and $[\mathbf{0}, \eta)$ is a fixed subinterval of $[0,1)^{d}$.

At this stage we mention the following condition, based on the base $q_{l}$ representation of $a^{(l, j)} / q_{l}^{d^{(l, j)}}=\left(0, a_{1}^{(l, j)} a_{2}^{(l, j)} \cdots a_{d^{(l, j)}}^{(l, j)}\right)_{q l}$ and on the weighted sums of digits related to the rows of the generating matrices, which will be used later. A point $\boldsymbol{x}_{n}$ lies in $J_{s}$ if and only if $n$ satisfies

$$
\begin{aligned}
s_{q_{l}, \gamma_{r}^{(l, j)}}(n) \equiv a_{r}^{(l, j)} \bmod q_{l}, & \\
& \quad \forall l \in\{1, \ldots, v\}, j \in\left\{1, \ldots, w_{l}\right\}, r \in\left\{1, \ldots, d^{(l, j)}\right\} .
\end{aligned}
$$

In [6, Proof of Theorem 4] the following exponential sum was used to decide whether $n$ satisfies (2.1) or not:

$$
\frac{1}{q_{1}} \sum_{z_{1}^{(1,1)}=0}^{q_{1}-1} \cdots \frac{1}{q_{1}} \sum_{z_{d^{(1,1)}}^{(1,1)}=0}^{q_{1}-1} \cdots \frac{1}{q_{v}} \sum_{z_{d^{(v, 1)}}^{(v, 1)}=0}^{q_{v}-1} \cdots \frac{1}{q_{v}} \sum_{z_{d^{\left(v, w_{v}\right)}}^{\left(v, w_{v}\right)}=0}^{q_{v}-1} \prod,
$$

where

$$
\prod:=\prod_{l=1}^{v} e\left(\sum_{j=1}^{w_{l}} \sum_{r=1}^{d^{(l, j)}}\left(\left(s_{q_{l}, \gamma_{r}^{(l, j)}}(n)-a_{r}^{(l, j)}\right) z_{r}^{(l, j)}\right) / q_{l}\right),
$$

where we redenote $e^{2 \pi \mathrm{i} x}$ by $e(x)$ here and later on. Note that this exponential sum equals 1 if $n$ satisfies (2.1) and 0 otherwise (for further details we refer the interested reader to [6]).

For the proof of uniform distribution we have to ensure that

$$
\lim _{N \rightarrow \infty} \frac{\#\left\{0 \leq n<N: z_{n} \in J\right\}}{N}=\lambda(J)=\lambda\left(J_{s}\right) \lambda\left(J_{d}\right) .
$$

From the assumption that the component sequences are uniformly distributed we know that

$$
\lim _{N \rightarrow \infty} \frac{\#\left\{0 \leq n<N: \boldsymbol{x}_{n} \in J_{s}\right\}}{N}=\lambda\left(J_{S}\right) .
$$

We define $M(N):=\left\{0 \leq n<N: \boldsymbol{x}_{n} \in J_{s}\right\}$ and $K(N):=|M(N)|$. Hence we have $\lim _{N \rightarrow \infty} K(N) / N=\lambda\left(J_{S}\right)$, and since $\lambda\left(J_{S}\right)>0$, we have $K(N)>0$ for sufficiently large $N$. Furthermore, the elements of $M(N)$ are, in increasing order, denoted by $0 \leq k_{0}<k_{1}<k_{2}<\cdots<k_{K(N)-1}$. 
We rewrite

$$
\frac{\#\left\{0 \leq n<N: z_{n} \in J\right\}}{N}=\frac{K(N)}{N} \frac{\#\left\{0 \leq m<K(N): \boldsymbol{y}_{k_{m}} \in J_{d}\right\}}{K(N)} .
$$

From the fact that $\lim _{N \rightarrow \infty} K(N) / N=\lambda\left(J_{S}\right)$ we see that it remains to be shown that

$$
\lim _{N \rightarrow \infty} \frac{\#\left\{0 \leq m<K(N): \boldsymbol{y}_{k_{m}} \in J_{d}\right\}}{K(N)}=\lambda\left(J_{d}\right) .
$$

To this end we prove that the subsequence $\left(\boldsymbol{y}_{k_{m}}\right)_{m \geq 0}$ is uniformly distributed modulo one and estimate the exponential sums that occur in Weyl's criterion. We fix $\boldsymbol{h} \in \mathbb{Z}^{d}$, $\boldsymbol{h} \neq \mathbf{0}$, and consider, for $N$ large enough such that $K(N)>0$,

$$
\begin{aligned}
& \frac{1}{K(N)} \sum_{m=0}^{K(N)-1} e\left(\boldsymbol{h} \cdot \boldsymbol{y}_{k_{m}}\right) \\
& =\frac{1}{K(N)} \sum_{m=0}^{K(N)-1} e\left(\boldsymbol{h} \cdot\left\{k_{m} \boldsymbol{\alpha}\right\}\right) \\
& =\frac{1}{K(N)} \sum_{m=0}^{K(N)-1} e\left((\boldsymbol{h} \cdot \boldsymbol{\alpha}) k_{m}\right) \\
& =\frac{N}{K(N)} \frac{1}{N} \sum_{n=0}^{N-1} e((\boldsymbol{h} \cdot \boldsymbol{\alpha}) n) \\
& \times \frac{1}{q_{1}} \sum_{z_{1}^{(1,1)}=0}^{q_{1}-1} \cdots \frac{1}{q_{1}} \sum_{z_{d^{(1,1)}}^{(1,1)}=0}^{q_{1}-1} \cdots \frac{1}{q_{v}} \sum_{z_{d^{(v, 1)}}^{(v, 1)}=0}^{q_{v}-1} \cdots \frac{1}{q_{v}} \sum_{z_{d^{\left(v, w_{v}\right)}}^{\left(v, w_{v}\right)}=0}^{q_{v}-1} \prod,
\end{aligned}
$$

where $\prod$ is defined as above.

In the latter expression, we exchange the summation order and prescind terms which are independent of $N$. Having done so, we see that we essentially need to deal with the following exponential sums depending on the values of $z_{r}^{(l, j)}$ (note that there are just finitely many different choices of the $\left.z_{r}^{(l, j)}\right)$ :

$$
\frac{1}{N} \sum_{n=0}^{N-1}\left(e((\boldsymbol{h} \cdot \boldsymbol{\alpha}) n) \prod_{l=1}^{v} e\left(\sum_{j=1}^{w_{l}} \sum_{r=1}^{d^{(l, j)}} z_{r}^{(l, j)} s_{q_{l}, \gamma_{r}^{(l, j)}}(n) / q_{l}\right)\right) .
$$

In order to conclude the proof of Theorem 2.1 it remains to prove that, for each admissible choice of the $z_{r}^{(l, j)}$, the sum (2.2) tends to zero as $N$ increases. We distinguish several cases.

Case 1. Assume first that all $z_{r}^{(l, j)}$ equal zero. Then the sum (2.2) reduces to $(1 / N) \sum_{n=0}^{N-1} e((\boldsymbol{h} \cdot \boldsymbol{\alpha}) n)$, which tends to zero as $N$ increases by using the assumption that $\left(\boldsymbol{y}_{n}\right)_{n \geq 0}$ is uniformly distributed and Weyl's criterion. 
Case 2. Suppose now that at least one of the $z_{r}^{(l, j)}$ is greater than zero. For each $l \in\{1, \ldots, v\}$, we introduce a sequence $\chi^{(l)}=\left(\chi_{k}^{(l)}\right)_{k \geq 0}$ with

$$
\chi_{k}^{(l)}:=\sum_{j=1}^{w_{l}} \sum_{r=1}^{d^{(l, j)}} z_{r}^{(l, j)} \gamma_{r, k}^{(l, j)} / q_{l} .
$$

Note that the elements of the sequence $\chi^{(l)}$ either are integers or have fractional parts contained in the set $\left\{1 / q_{l}, \ldots,\left(q_{l}-1\right) / q_{l}\right\}$.

We observe that $f(n):=(\boldsymbol{h} \cdot \boldsymbol{\alpha}) n$ is $q$-additive in any base $q$, since it is even additive (that is, $f(a+b)=f(a)+f(b)$ for all integers $a, b \geq 0)$. We choose a prime $q_{v+1}$ that is different from $q_{1}, \ldots, q_{v}$ and regard $f$ as a $q_{v+1}$-additive function.

Furthermore,

$$
\sum_{j=1}^{w_{l}} \sum_{r=1}^{d^{(l, j)}} z_{r}^{(l, j)} s_{q_{l}, \gamma_{r}^{(l, j)}}(n) / q_{l}=: g^{(l)}(n)
$$

is a $q_{l}$-additive function because the weighted sums of digits are all in base $q_{l}$. Hence we can write (2.2) as

$$
\frac{1}{N} \sum_{n=0}^{N-1}\left(e(f(n)) \prod_{l=1}^{v} e\left(g^{(l)}(n)\right)\right),
$$

where each of the functions $f, g^{(1)}, \ldots, g^{(v)}$ is additive with respect to a different prime.

We distinguish two subcases.

Case 2.1. Suppose first that for each $l$ the sequence $\chi^{(l)}$ contains only finitely many entries that are not integers. In this case the value of the term $\prod_{l=1}^{v} g^{(l)}(n)$ depends only on finitely many digits of the base $q_{l}$ representations of $n$, say the first $b_{l}$, for all $l$. We partition the set $\mathbb{N}_{0}$ into the residue classes modulo $q_{1}^{b_{1}} \cdots q_{l}^{b_{l}}=: \mu$. Let $0 \leq \rho<q_{1}^{b_{1}} \cdots q_{l}^{b_{l}}$ be fixed. We consider the exponential sum

$$
\begin{aligned}
\lim _{N \rightarrow \infty} & \frac{1}{N} \sum_{\substack{n=0 \\
n \equiv \rho(\bmod ) \mu}}^{N-1}\left(e((\boldsymbol{h} \cdot \boldsymbol{\alpha}) n) \prod_{l=1}^{v} e\left(g^{(l)}(\rho)\right)\right) \\
= & \lim _{N \rightarrow \infty} C(\rho, \alpha, \boldsymbol{h}) \frac{1}{N} \sum_{n=0}^{\lfloor(N-1-\rho) / \mu\rfloor} e((\boldsymbol{h} \cdot \mu \boldsymbol{\alpha}) n)
\end{aligned}
$$

where $C(\rho, \alpha, \boldsymbol{h})$ is a constant independent of $n$. From our assumption that $\left(\boldsymbol{y}_{n}\right)_{n \geq 0}$ is uniformly distributed we know that $\boldsymbol{h} \cdot \boldsymbol{\alpha}$ is irrational and hence $\boldsymbol{h} \cdot \boldsymbol{\mu} \boldsymbol{\alpha}$ is also irrational. This implies that the latter limit equals zero (see, for example, [11, p. 8]). As this holds for any admissible choice of $\rho$, the result follows.

Case 2.2. Assume, finally, that for at least one $l$ the corresponding sequence $\chi^{(l)}$ contains infinitely many entries that are not integers. We choose such an $l$ and examine 
the parameter $\tau_{k}^{(l)}$ in Proposition 2.2. We have

$$
\theta_{k}^{(l)}(a)=g^{(l)}\left((a+1) q_{l}^{k}\right)-g^{(l)}\left(a q_{l}^{k}\right)-g^{(l)}\left(q_{l}^{k}\right)=\chi_{k}^{(l)}(a+1)-\chi_{k}^{(l)} a-\chi_{k}^{(l)}=0 .
$$

Hence

$$
\tau_{k}^{(l)}=\left\|g^{(l)}\left(q_{l}^{k+1}\right)-q_{l} g^{(l)}\left(q_{l}^{k}\right)\right\|^{2} / 4=\left\|\chi_{k+1}^{(l)}\right\|^{2} / 4
$$

Note that if $\chi_{k+1}^{(l)} \notin \mathbb{Z}$ then $\left\|\chi_{k+1}^{(l)}\right\|^{2} / 4 \geq 1 /\left(4 q_{l}^{2}\right)$. Since we assumed infinitely many elements in the sequence $\chi^{(l)}$ to be noninteger, we obtain $\sum_{k=0}^{\infty} \tau_{k}^{(l)}=\infty$, and invoking Proposition 2.2 we see that the exponential sum (2.2) tends to zero as $N$ increases.

The proof of Theorem 2.1 is mainly based on the distribution properties of special subsequences of a Kronecker sequence and yields the following corollary.

COROLlary 2.3. Let $\boldsymbol{\alpha} \in[0,1)^{d}$ and let $(\{n \boldsymbol{\alpha}\})_{n \geq 0}$ be the corresponding Kronecker sequence. Let $\left(k_{n}\right)_{n \geq 0}$ be the increasing sequence of nonnegative integers obtained by solving the following system of equations. For $v \in \mathbb{N}$ let $q_{1}, \ldots, q_{v}$ be different primes and let $y_{1}, \ldots, y_{v}$ be positive integers. For every pair $(i, k)$ with $i \in\{1, \ldots, v\}$, $k \in\left\{1, \ldots, y_{v}\right\}$, we consider

$$
\left(\rho_{0}^{(i, k)}, \rho_{1}^{(i, k)}, \ldots\right) \cdot\left(x_{0}^{(i)}, x_{1}^{(i)}, \ldots\right)^{T}=b^{(i, k)}
$$

over $\mathbb{Z}_{q_{i}}^{\mathbb{N}_{0}}$, with fixed $b^{(i, k)} \in \mathbb{Z}_{q_{i}}$ and fixed $\rho_{h}^{(i, k)} \in \mathbb{Z}_{q_{i}}$ for all $h \geq 0$. We call $a$ nonnegative integer $n$ a solution of this system of equations if, for each $i \in\{1, \ldots, v\}$, the digit-vector $\left(n_{0}^{(i)}, n_{1}^{(i)}, \ldots\right)^{T}$ corresponding to the base $q_{i}$ representation of $n=n_{0}^{(i)}+n_{1}^{(i)} q_{i}+\cdots$ solves all the $y_{i}$ equations over $\mathbb{Z}_{q_{i}}^{\mathbb{N}_{0}}$. If the system of equations is solvable, then the subsequence $\left(\left\{k_{n} \alpha\right\}\right)_{n \geq 0}$ obtained by the infinitely many solutions is uniformly distributed if and only if the sequence $(\{n \alpha\})_{n \geq 0}$ is uniformly distributed.

The method of proof used for Theorem 2.1 can also be applied to other hybrid sequences, as the following example shows. Let $q_{1}, \ldots, q_{v}$ be different primes. Moreover, let $\left(\alpha_{1}, \ldots, \alpha_{d}\right):=\alpha \in[0,1)^{d}$ and $p_{1}, \ldots, p_{d}$ be integers greater than or equal to two, which are pairwise either equal or coprime, and also pairwise coprime with $q_{1}, \ldots, q_{v}$. For $n \geq 0$, we define $\boldsymbol{w}_{n}:=\left(\left\{\alpha_{1} s_{p_{1}}(n)\right\}, \ldots,\left\{\alpha_{d} s_{p_{d}}(n)\right\}\right)$, where $s_{p_{i}}(n)$ denotes the nonweighted sum of digits in base $p_{i}$, that is, $s_{p_{i}}(n)=s_{p_{i},(1)_{k \geq 0}}(n)$. We can then derive the uniform distribution of the sequence $\left(\boldsymbol{x}_{n}, \boldsymbol{w}_{n}\right)_{n \geq 0}$, where $\left(\boldsymbol{x}_{n}\right)_{n \geq 0}$ is a uniformly distributed Niederreiter-Halton sequence in bases $q_{1}, \ldots, q_{v}$ as in Definition 1.5, and where $\left(\boldsymbol{w}_{n}\right)_{n \geq 0}$ is uniformly distributed. The distribution of the sequence $\left(\boldsymbol{w}_{n}\right)_{n \geq 0}$ was investigated in [2]. To show the uniform distribution of this hybrid sequence, we can apply the method of proof of Theorem 2.1, and the problem reduces to dealing with a term of the form

$$
\lim _{N \rightarrow \infty} \frac{1}{N} \sum_{n=0}^{N-1} \prod_{i=1}^{d}\left(e\left(h_{i} \alpha_{i} s_{p_{i}}(n)\right)\right) \prod_{l=1}^{v} e\left(\sum_{j=1}^{w_{l}} \sum_{r=1}^{d^{(l, j)}} z_{r}^{(l, j)} s_{q_{l}, \gamma_{r}^{(l, j)}}(n) / q_{l}\right) .
$$


Again, we can use Proposition 2.2 to show that this limit is zero and deduce uniform distribution of $\left(\boldsymbol{x}_{n}, \boldsymbol{w}_{n}\right)_{n \geq 0}$. Note that this method could be further generalized to hybrid sequences made up of those considered in [4, 19] and of Niederreiter-Halton sequences.

\section{Discrepancy estimates}

As we have seen in Section 2, a hybrid sequence obtained by mixing a NiederreiterHalton sequence and a Kronecker sequence is uniformly distributed whenever its component sequences are uniformly distributed. This is a qualitative result. Needless to say, we are also interested in quantitative results, that is, we would like to have information about the quality of the distribution of a sequence. One way of measuring the extent to which a sequence is uniformly distributed is to consider its discrepancy. In order to define discrepancy, we first define a local discrepancy function.

For given $N \geq 1$, a point set $P$ of $N$ points in $[0,1)^{s}$, and an interval $I \subseteq[0,1)^{s}$, we write

$$
\Delta(P, I):=A_{N}(P, I)-N \lambda(I)
$$

for the local discrepancy function, where $A_{N}(P, I)$ denotes the number of points of $P$ in $I$, and $\lambda$ denotes the ( $s$-dimensional) Lebesgue measure.

Using the function $\Delta$, we now define the discrepancy, which is sometimes also referred to as extreme discrepancy, of a point set.

Definition 3.1. For given $N \geq 1$, and a point set $P$ of $N$ points in $[0,1)^{s}$, we define the discrepancy of $P$ by

$$
D_{N}(P):=\sup _{I \subseteq[0,1)^{s}}\left|\frac{\Delta(P, I)}{N}\right|,
$$

where $\Delta$ is defined as above. For an infinite sequence $S, D_{N}(S)$ denotes the discrepancy of the first $N$ points of $S$.

It is well known that a sequence $S$ is uniformly distributed modulo one if and only if $D_{N}(S)$ tends to zero as $N$ increases. Therefore, knowledge of how fast this convergence takes place is valuable, that is, one is interested in discrepancy bounds. One classical result with respect to discrepancy bounds is the Erdős-Turán-Koksma inequality. See, for example, [1, 3, 11], for further details.

THEOREM 3.2 (Erdős-Turán-Koksma inequality). For given $N \geq 1$ and a point set $P$ of $N$ points $\boldsymbol{x}_{0}, \ldots, \boldsymbol{x}_{N-1}$ in $[0,1)^{s}$, the inequality

$$
D_{N}(P) \leq\left(\frac{3}{2}\right)^{s}\left(\frac{2}{m+1}+\sum_{\substack{\boldsymbol{h} \in \mathbb{Z}^{d} \\ 0<\|\boldsymbol{h}\|_{\infty} \leq m}} \frac{1}{r(\boldsymbol{h})}\left|\frac{1}{N} \sum_{n=0}^{N-1} e\left(\boldsymbol{h} \cdot \boldsymbol{x}_{n}\right)\right|\right)
$$

holds, where $m$ is an arbitrarily chosen positive integer, $\|\boldsymbol{h}\|_{\infty}:=\max _{1 \leq j \leq d}\left|h_{j}\right|$, and $r(\boldsymbol{h}):=\prod_{j=1}^{d} \max \left\{1,\left|h_{j}\right|\right\}$. 
Apart from the extreme discrepancy, one frequently also considers the star discrepancy of a point set, which is defined as follows.

Definition 3.3. For given $N \geq 1$, and a point set $P$ of $N$ points in $[0,1)^{s}$, we define the star discrepancy of $P$ by

$$
D_{N}^{*}(P):=\sup _{I^{*} \subseteq[0,1)^{s}}\left|\frac{\Delta\left(P, I^{*}\right)}{N}\right|,
$$

where $\Delta$ is defined as above, and where the supremum is extended over all intervals $I^{*} \subseteq[0,1)^{s}$ with lower left corner at the origin. For an infinite sequence $S, D_{N}^{*}(S)$ denotes the star discrepancy of the first $N$ points of $S$.

It is well known in the theory of uniform distribution (see, for example, [11]) that the extreme and the star discrepancy of a given $s$-dimensional point set can be bounded by means of each other,

$$
D_{N}^{*} \leq D_{N} \leq 2^{s} D_{N}^{*}
$$

Hence, the Erdôs-Turán-Koksma inequality trivially also holds for the star discrepancy.

For classical point sets, such as $(\mathbf{T}, s)$-sequences or Kronecker sequences, many discrepancy bounds are known (see, for example, [1, 3, 11, 15, 16]). Accordingly, an obvious question regarding the class of hybrid sequences studied in Section 2 is whether it is possible to give discrepancy bounds for these point sets. In this section we show such results for special cases.

To make the notation easier, we use the following convention throughout the rest of the paper. We say that a number $x \in[0,1)$ is $m$-bit (in base $q \geq 2$ ), for $m \geq 1$, if $x$ is of the form

$$
x=\sum_{r=1}^{m} \frac{x_{r}}{q^{r}}, \quad x_{r} \in\{0,1, \ldots, q-1\} .
$$

Assume that we are given an $s$-dimensional Niederreiter-Halton sequence $S=$ $\left(\boldsymbol{x}_{n}\right)_{n \geq 0}$ as in Definition 1.5. Furthermore, assume that there exist constant integers $K_{l, j}, l \in\{1, \ldots, v\}, j \in\left\{1, \ldots, w_{l}\right\}$, such that the elements in the rows of the generating matrices of $S$ satisfy

$$
\gamma_{r, k}^{(l, j)}=0 \quad \text { if } k \geq r+K_{l, j}, \forall l \in\{1, \ldots, v\}, j \in\left\{1, \ldots, w_{l}\right\} .
$$

If (3.1) holds, we say that the generating matrices of $S$ are bounded over the diagonal. If $w_{l}=1$ we write, for the sake of simplicity, $K_{l}$ for the constants $K_{l, 1}$ in condition (3.1).

We now show the following proposition, which is inspired by [8, Proposition 1].

Proposition 3.4. Let $v \geq 1$ and let $q_{1}, \ldots, q_{v}$ be different primes. Assume that we are given an $s$-dimensional Niederreiter-Halton sequence $S_{1}=\left(x_{n}\right)_{n \geq 0}=$ $\left(x_{n}^{(1)}, \ldots, x_{n}^{(s)}\right)_{n \geq 0}$ with $w_{1}=\cdots=w_{v}=1$ (that is, $s=v$ ). Furthermore, assume that the generating matrices of $S$ are bounded over the diagonal. 
Assume also that, for each $l \in\{1, \ldots, s\}$ and each $r \geq 1$, the row vectors $\gamma_{1}^{(l, 1)}, \ldots, \gamma_{r}^{(l, 1)}$ are linearly independent over $\mathbb{Z}_{q_{l}}$.

Let $S_{2}=\left(\boldsymbol{y}_{n}\right)_{n \geq 0}=(\{n \boldsymbol{\alpha}\})_{n \geq 0}, \quad \boldsymbol{\alpha} \in[0,1)^{d}$, be a d-dimensional uniformly distributed Kronecker sequence. Then the hybrid sequence $S=\left(z_{n}\right)_{n \geq 0}:=$ $\left(\left(\boldsymbol{x}_{n}, \boldsymbol{y}_{n}\right)\right)_{n \geq 0}$ satisfies the following upper bound on its star discrepancy:

$$
N D_{N}^{*}(S) \leq C \sum_{j_{1}=1}^{m_{1}-K_{1}} \cdots \sum_{j_{s}=1}^{m_{s}-K_{s}} \sum_{\substack{\boldsymbol{h} \in \mathbb{Z}^{d} \\ 0<\|\boldsymbol{h}\|_{\infty} \leq N}} \frac{1}{r(\boldsymbol{h})} \frac{1}{\left\|\prod_{l=1}^{s} q_{l}^{j_{l}+K_{l}} \boldsymbol{h} \cdot \boldsymbol{\alpha}\right\|}+\mathcal{O}\left((\log N)^{s}\right),
$$

where $C>0$ is a constant independent of $N, m_{l}=\left\lceil\log _{q_{l}} N\right\rceil, 1 \leq l \leq s$, and $\|\cdot\|$ denotes the distance to the nearest integer.

PROOF. First of all, we note that, due to the assumptions made, our sequence $S$ is uniformly distributed modulo one. This follows by combining results in [6] and the results in Section 2.

Now let $[\mathbf{0}, \boldsymbol{\beta}) \times[\mathbf{0}, \boldsymbol{\eta}) \subseteq[0,1)^{s+d}$ with

$$
\boldsymbol{\beta}=\left(\beta_{1}, \ldots, \beta_{s}\right), \quad \boldsymbol{\eta}=\left(\eta_{1}, \ldots, \eta_{d}\right) .
$$

Let $S$ be defined as above and let $S_{N}:=\left(z_{n}\right)_{n=0}^{N-1}$. We are aiming for a bound on

$$
\Delta\left(S_{N},[\mathbf{0}, \boldsymbol{\beta}) \times[\mathbf{0}, \boldsymbol{\eta})\right) .
$$

For each $l \in\{1, \ldots, s\}$, choose $m_{l}$ as the minimal integer such that $N \leq q_{l}^{m_{l}}$. For the rest of the proof, we assume that $N$ is large enough such that $m_{l}>K_{l}$ for all $l \in$ $\{1, \ldots, v\}$. The result follows easily for smaller $N$ by adjusting the constants in (3.2).

We now define

$$
\widetilde{S}_{N}:=\left(\widetilde{\boldsymbol{z}}_{n}\right)_{n \geq 0}:=\left(\left(\widetilde{\boldsymbol{x}}_{n}, \boldsymbol{y}_{n}\right)\right)_{n \geq 0}=\left(\left(\left(\widetilde{x}_{n}^{(1)}, \ldots, \widetilde{x}_{n}^{(s)}\right), \boldsymbol{y}_{n}\right)\right)_{n \geq 0},
$$

where each $\tilde{x}_{n}^{(l)}$ consists of the first $\left(m_{l}-K_{l}\right)$ bits of $x_{n}^{(l)}$ in the corresponding base $q_{l}$ representation, and is therefore, with respect to $q_{l}$, an $\left(m_{l}-K_{l}\right)$-bit number. Since, in the transition from $S_{N}$ to $\widetilde{S}_{N}$, we changed the values of the points by at most $q_{l}^{K_{l}} /\left(q_{l}^{m_{l}}\right)$ for the $l$ th component, straightforward arguments (see also [1, Proposition 3.15]) lead to

$$
\left|N D_{N}^{*}\left(S_{N}\right)-N D_{N}^{*}\left(\widetilde{S}_{N}\right)\right| \leq c_{1},
$$

where $c_{1}$ is a constant that might depend on $s, K_{1}, \ldots, K_{s}$, and $q_{1}, \ldots, q_{s}$, but does not depend on $N$. Hence it is sufficient to consider $\widetilde{S}_{N}$ instead of $S_{N}$ in the following.

On the other hand, from the structure of $\widetilde{S}_{N}$ and the fact that $N \leq q_{l}^{m_{l}}$ for all $l \in$ $\{1, \ldots, s\}$, it is easily deduced that

$$
\left|\Delta\left(\widetilde{S}_{N},[\mathbf{0}, \boldsymbol{\beta}) \times[\mathbf{0}, \boldsymbol{\eta})\right)-\Delta\left(\widetilde{S}_{N},[\mathbf{0}, \widetilde{\boldsymbol{\beta}}) \times[\mathbf{0}, \boldsymbol{\eta})\right)\right| \leq c_{2},
$$

where

$$
\widetilde{\boldsymbol{\beta}}=\left(\widetilde{\beta}_{1}, \ldots, \widetilde{\beta}_{s}\right),
$$


each of the $\widetilde{\beta}_{l}$ consisting of the first $m_{l}-K_{l}$ bits of $\beta_{l}$. The constant $c_{2}$ in the above inequality might depend on $s$, the $K_{l}$, and the $q_{l}$, but is independent of $N$, since the sequence $S$ is uniformly distributed modulo one. Indeed, the volume of the interval as well as the number of points we might lose by restricting ourselves to $\widetilde{\boldsymbol{\beta}}$ can be bounded independently of $N$, due to the fact that we chose the $m_{l}$ such that $N \leq q^{m_{l}}$. We refer the reader to $[7,8]$ for further details.

Hence we study

$$
\Delta\left(\widetilde{S}_{N},[\mathbf{0}, \widetilde{\boldsymbol{\beta}}) \times[\mathbf{0}, \boldsymbol{\eta})\right)
$$

in the following. We write the interval $I:=[\mathbf{0}, \widetilde{\boldsymbol{\beta}}) \times[\mathbf{0}, \boldsymbol{\eta})$ as the disjoint union of intervals

$$
\begin{aligned}
& \tilde{I}\left(j_{1}, \ldots, j_{s}, \boldsymbol{\eta}\right) \\
& \quad:=\prod_{l=1}^{s}\left[\sum_{r=1}^{j_{l}-1} \frac{\beta_{l}^{(r)}}{q_{l}^{r}}, \sum_{r=1}^{j_{l}} \frac{\beta_{l}^{(r)}}{q_{l}^{r}}\right) \times[\mathbf{0}, \boldsymbol{\eta}), 1 \leq j_{l} \leq m_{l}-K_{l}, \forall l \in\{1, \ldots, s\} .
\end{aligned}
$$

Note that each $\widetilde{I}\left(j_{1}, \ldots, j_{s}, \eta\right)$ can again be written as the disjoint union of intervals

$$
\prod_{l=1}^{s}\left[\sum_{r=1}^{j_{l}-1} \frac{\beta_{l}^{(r)}}{q_{l}^{r}}+\frac{k_{l}}{q_{l}^{j_{l}}}, \sum_{r=1}^{j_{l}-1} \frac{\beta_{l}^{(r)}}{q_{l}^{r}}+\frac{k_{l}+1}{q_{l}^{j_{l}}}\right) \times[\mathbf{0}, \boldsymbol{\eta}),
$$

with $1 \leq j_{l} \leq m_{l}-K_{l}, 0 \leq k_{l} \leq \beta_{l}^{\left(j_{l}\right)}-1$.

Now let $1 \leq l \leq s$ be fixed. By the construction principle of $\left(\widetilde{x}_{n}^{(l)}\right)_{n \geq 0}$, we see that

$$
\tilde{x}_{n}^{(l)} \in\left[\sum_{r=1}^{j_{l}-1} \frac{\beta_{l}^{(r)}}{q_{l}^{r}}+\frac{k_{l}}{q_{l}^{j_{l}}}, \sum_{r=1}^{j_{l}-1} \frac{\beta_{l}^{(r)}}{q_{l}^{r}}+\frac{k_{l}+1}{q_{l}^{j_{l}}}\right)=: J\left(j_{l}, k_{l}\right)
$$

if and only if

$$
\left(\begin{array}{ccc}
\gamma_{1,0}^{(l, 1)} & \ldots & \gamma_{1, j_{l}+K_{l}-1}^{(l, 1)} \\
\gamma_{2,0}^{(l, 1)} & \ldots & \gamma_{2, j_{l}+K_{l}-1}^{(l, 1)} \\
\vdots & & \vdots \\
\gamma_{j_{l}, 0}^{(l, 1)} & \ldots & \gamma_{j_{l}, j_{l}+K_{l}-1}^{(l, 1)}
\end{array}\right) \cdot\left(\begin{array}{c}
n_{0}^{(l)} \\
n_{1}^{(l)} \\
\vdots \\
n_{j_{l}+K_{l}-1}^{(l)}
\end{array}\right)=\left(\begin{array}{c}
\beta_{l}^{(1)} \\
\beta_{l}^{(2)} \\
\vdots \\
\beta_{l}^{\left(j_{l}-1\right)} \\
k_{l}
\end{array}\right)
$$

where the numbers $n_{i}^{(l)}$ are the digits of $n$ in base $q_{l}$. Due to our assumptions on the regularity of the rows $\gamma_{1}^{(l, 1)}, \ldots, \gamma_{j l}^{(l, 1)}$, the system (3.3) has exactly $q_{l}^{K_{l}}$ solutions over $\mathbb{Z}_{q_{l}}$.

Hence we can identify $q_{l}^{K_{l}}$ remainders $R_{i}^{(l)}, i \in\left\{1, \ldots, q_{l}^{K_{l}}\right\}$, modulo $q_{l}^{j_{l}+K_{l}}$ such that $\tilde{x}_{n}^{(l)} \in J\left(j_{l}, k_{l}\right)$ if and only if $n \equiv R_{i}^{(l)} \bmod q_{l}^{j_{l}+K_{l}}$ for an $i \in\left\{1, \ldots, q_{l}^{K_{l}}\right\}$.

Invoking the Chinese remainder theorem, there exist $\prod_{l=1}^{s} q_{l}^{K_{l}}=: K$ remainders $R_{1}, \ldots, R_{K}$ modulo $Q\left(j_{1}, \ldots, j_{s}\right)=Q:=\prod_{l=1}^{s} q_{l}^{j_{l}+K_{l}}$ such that $\widetilde{\boldsymbol{x}}_{n} \in \prod_{l=1}^{s} J\left(j_{l}, k_{l}\right)$ if and only if $n \equiv R_{i} \bmod Q$ for one $i \in\{1, \ldots, K\}$. 
We now deduce an estimate for

$$
\left|\Delta\left(\widetilde{S}_{N}, \prod_{l=1}^{s} J\left(j_{l}, k_{l}\right) \times[\mathbf{0}, \boldsymbol{\eta})\right)\right| .
$$

The Lebesgue measure of such an interval is $\eta_{1} \cdots \eta_{d} /\left(q_{1}^{j_{1}} \cdots q_{s}^{j_{s}}\right)$ and, as already noted, $z_{n}$ is included in such an interval if and only if $n \equiv R_{i} \bmod Q$ for one $i \in$ $\{1, \ldots, K\}$ and $\{n \boldsymbol{\alpha}\} \in[\mathbf{0}, \boldsymbol{\eta})$. The first of these conditions is fulfilled if and only if $n$ is contained in one of the disjoint sets

$$
\left\{u Q+R_{i}: 0 \leq u \leq U_{i}\left(Q, R_{i}\right)\right\},
$$

where $U_{i}\left(Q, R_{i}\right):=\lfloor N / Q\rfloor+\theta_{i}$, for a certain $\theta_{i} \in\{0,1\}$. Altogether we obtain

$$
\left|\Delta\left(\tilde{S}_{N}, \prod_{l=1}^{s} J\left(j_{l}, k_{l}\right) \times[\mathbf{0}, \boldsymbol{\eta})\right)\right| \leq 2 K+\sum_{i=1}^{K} U_{i}\left(Q, R_{i}\right) D_{U_{i}\left(Q, R_{i}\right)}\left(S_{2}^{(i)}\right),
$$

where, for $1 \leq i \leq K, S_{2}^{(i)}:=\left(\left\{\left(u Q+R_{i}\right) \alpha\right\}\right)_{u \geq 0}$. Now we use the Erdős-TuránKoksma inequality to obtain

$$
\begin{aligned}
& U_{i}\left(Q, R_{i}\right) D_{U_{i}\left(Q, R_{i}\right)}\left(S_{2}^{(i)}\right) \\
& \quad \leq\left(\frac{3}{2}\right)^{d} \frac{U_{i}\left(Q, R_{i}\right)}{N+1}+\left(\frac{3}{2}\right)^{d} \sum_{\substack{\boldsymbol{h} \in \mathbb{Z}^{d} \\
0<\|\boldsymbol{h}\|_{\infty} \leq N}} \frac{1}{r(\boldsymbol{h})}\left|\sum_{u=0}^{U_{i}\left(Q, R_{i}\right)-1} e\left(\boldsymbol{h} \cdot\left\{\left(u Q+R_{i}\right) \boldsymbol{\alpha}\right\}\right)\right| \\
& \quad \leq c_{3}+c_{4} \sum_{\substack{\boldsymbol{h} \in \mathbb{Z}^{d} \\
0<\|\boldsymbol{h}\|_{\infty} \leq N}} \frac{1}{r(\boldsymbol{h})}\left|\sum_{u=0}^{U_{i}\left(Q, R_{i}\right)-1} e\left(\boldsymbol{h} \cdot\left\{\left(u Q+R_{i}\right) \boldsymbol{\alpha}\right\}\right)\right|,
\end{aligned}
$$

where $c_{3}, c_{4}$ are positive constants independent of $N$. Using methods similar to those in [14, 17], we obtain

$$
\begin{aligned}
\left|\sum_{u=0}^{U_{i}\left(Q, R_{i}\right)-1} e\left(\boldsymbol{h} \cdot\left\{\left(u Q+R_{i}\right) \boldsymbol{\alpha}\right\}\right)\right| & =\left|\sum_{u=0}^{U_{i}\left(Q, R_{i}\right)-1} e\left(\boldsymbol{h} \cdot\left(\left(u Q+R_{i}\right) \boldsymbol{\alpha}\right)\right)\right| \\
& =\left|\sum_{u=0}^{U_{i}\left(Q, R_{i}\right)-1} e(u(\boldsymbol{h} \cdot Q \boldsymbol{\alpha}))\right| \\
& \leq \frac{2}{|e(\boldsymbol{h} \cdot Q \boldsymbol{\alpha})-1|} \\
& =\frac{1}{\sin (\pi\|\boldsymbol{h} \cdot Q \boldsymbol{\alpha}\|)} \\
& \leq \frac{1}{2\|Q \boldsymbol{h} \cdot \boldsymbol{\alpha}\|},
\end{aligned}
$$

where, as above, $\|\cdot\|$ denotes the distance to the nearest integer. 
Summing up, we obtain

$$
\begin{aligned}
\left|\Delta\left(S_{N},[\mathbf{0}, \boldsymbol{\beta}) \times[\mathbf{0}, \boldsymbol{\eta})\right)\right| & \leq c_{1}+c_{2}+\left|\Delta\left(\widetilde{S}_{N},[\mathbf{0}, \widetilde{\boldsymbol{\beta}}) \times[\mathbf{0}, \boldsymbol{\eta})\right)\right| \\
\leq & c_{1}+c_{2}+\sum_{j_{1}=1}^{m_{1}-K_{1}} \cdots \sum_{j_{s}=1}^{m_{s}-K_{s}} K \\
& \times\left(2+c_{3}+c_{4} \sum_{\substack{\boldsymbol{h} \in \mathbb{Z}^{d} \\
0<\|\boldsymbol{h}\|_{\infty} \leq N}} \frac{1}{r(\boldsymbol{h})} \frac{1}{2\left\|\prod_{l=1}^{s} q_{l}^{j_{l}+K_{l}} \boldsymbol{h} \cdot \boldsymbol{\alpha}\right\|}\right) \\
= & C \sum_{j_{1}=1}^{m_{1}-K_{1}} \cdots \sum_{j_{s}=1}^{m_{s}-K_{s}} \sum_{\substack{\boldsymbol{h} \in \mathbb{Z}^{d} \\
0<\|\boldsymbol{h}\|_{\infty} \leq N}} \frac{1}{r(\boldsymbol{h})} \frac{1}{\left\|\prod_{l=1}^{s} q_{l}^{j_{l}+K_{l}} \boldsymbol{h} \cdot \boldsymbol{\alpha}\right\|}+\mathcal{O}\left((\log N)^{s}\right),
\end{aligned}
$$

with $C>0$ independent of $N$, as claimed.

From Proposition 3.4, we derive the following theorem, which is an analogue of [8, Theorem 1].

Theorem 3.5. Let $S_{1}=\left(\boldsymbol{x}_{n}\right)_{n \geq 0}$ be defined as in Proposition 3.4. Then for almost all $\boldsymbol{\alpha} \in[0,1)^{d}$ the sequence $S=\left(\boldsymbol{z}_{n}\right)_{n \geq 0}:=\left(\left(\boldsymbol{x}_{n}, \boldsymbol{y}_{n}\right)\right)_{n \geq 0}$, where $S_{2}=\left(\boldsymbol{y}_{n}\right)_{n \geq 0}=$ $(\{n \boldsymbol{\alpha}\})_{n \geq 0}$ is the d-dimensional Kronecker sequence corresponding to $\boldsymbol{\alpha}$, satisfies, for every $\varepsilon>0$,

$$
N D_{N}^{*}(S)=\mathcal{O}\left((\log N)^{s+d+1+\varepsilon}\right)
$$

with the implied constant independent of $N$.

PROOF. Using Proposition 3.4,

$$
\begin{aligned}
N D_{N}^{*}(S) & \leq C \sum_{j_{1}=1}^{m_{1}-K_{1}} \cdots \sum_{j_{s}=1}^{m_{s}-K_{s}} \sum_{\substack{\boldsymbol{h} \in \mathbb{Z}^{d} \\
0<\|\boldsymbol{h}\|_{\infty} \leq N}} \frac{1}{r(\boldsymbol{h})} \frac{1}{\left\|\prod_{l=1}^{s} q_{l}^{j_{l}+K_{l}} \boldsymbol{h} \cdot \boldsymbol{\alpha}\right\|}+\mathcal{O}\left((\log N)^{s}\right) \\
& \leq C \sum_{j_{1}=1}^{m_{1}} \cdots \sum_{j_{s}=1}^{m_{s}} \sum_{\substack{\boldsymbol{h} \in \mathbb{Z}^{d} \\
0<\|\boldsymbol{h}\|_{\infty} \leq N}} \frac{1}{r(\boldsymbol{h})} \frac{1}{\left\|\prod_{l=1}^{s} q_{l}^{j_{l}} \boldsymbol{h} \cdot \boldsymbol{\alpha}\right\|}+\mathcal{O}\left((\log N)^{s}\right) .
\end{aligned}
$$

In the latter expression, we can separate out those components of $\boldsymbol{h}$ which are different from zero and then make use of [8, Proposition 3], which yields the result.

\section{Conclusion and outlook}

In this paper, we have discussed necessary and sufficient conditions for uniform distribution of hybrid sequences that are built from Niederreiter-Halton and Kronecker sequences. We have shown in Theorem 2.1 that combining a uniformly distributed 
Niederreiter-Halton sequence and a uniformly distributed Kronecker sequence automatically yields a uniformly distributed hybrid sequence. Regarding discrepancy, Theorem 3.5 gives a probabilistic result. However, we need to assume that the generating matrices of the Niederreiter-Halton sequence involved are bounded over the diagonal. An obvious question is whether or to what extent this condition on the generating matrices can be relaxed, and which discrepancy bounds then hold.

As an open question for future research we pose the following problem.

QUESTION 4.1. Let $S_{1}=\left(x_{n}\right)_{n \geq 0}$ be a one-dimensional sequence, which is generated by the matrix

$$
\left(\begin{array}{cccccc}
1 & 1 & 1 & 1 & 1 & \ldots \\
0 & 1 & 0 & 0 & 0 & \ldots \\
0 & 0 & 1 & 0 & 0 & \ldots \\
0 & 0 & 0 & 1 & 0 & \ldots \\
0 & 0 & 0 & 0 & 1 & \ldots \\
\vdots & \vdots & \vdots & \vdots & \vdots & \ddots
\end{array}\right)
$$

over $\mathbb{Z}_{2}$. Furthermore, let $S_{2}=\left(y_{n}\right)_{n \geq 0}=(\{n \alpha\})_{n \geq 0}$ be a one-dimensional Kronecker sequence. Do there exist any $\alpha$ such that the sequence $S=\left(\left(x_{n}, y_{n}\right)\right)_{n \geq 0}$ satisfies a discrepancy bound like the one in Theorem 3.5? If so, what is the Lebesgue measure of the set of such $\alpha$ ? To answer this question we would at least need good lower and upper bounds for the discrepancy of the subsequence $\left(\left\{k_{n} \alpha\right\}\right)_{n \geq 0}$, where the increasing sequence $\left(k_{n}\right)_{n \geq 0}$ runs through all nonnegative integers with an even sum of digits in base 2 .

\section{Acknowledgement}

The authors would like to thank Gerhard Larcher for valuable discussions and comments.

\section{References}

[1] J. Dick and F. Pillichshammer, Digital Nets and Sequences. Discrepancy Theory and Quasi-Monte Carlo Integration (Cambridge University Press, Cambridge, 2010).

[2] M. Drmota and G. Larcher, 'The sum-of-digits function and uniform distribution modulo 1', J. Number Theory 89 (2001), 65-96.

[3] M. Drmota and R. F. Tichy, Sequences, Discrepancies and Applications, Lecture Notes in Mathematics, 1651 (Springer, Berlin, 1997).

[4] R. Hofer, 'Note on the joint distribution of the weighted sum-of-digits function modulo one in case of pairwise coprime bases', Unif. Distrib. Theory 2 (2007), 35-47.

[5] R. Hofer, 'On subsequences of Niederreiter-Halton sequences', in: Monte Carlo and Quasi-Monte Carlo Methods 2008 (eds. P. L’Ecuyer and B. Owen) (Springer, Berlin, 2009), pp. 423-438.

[6] R. Hofer, 'On the distribution properties of Niederreiter-Halton sequences', J. Number Theory 129 (2009), 451-463.

[7] R. Hofer, P. Kritzer, G. Larcher and F. Pillichshammer, 'Distribution properties of generalized van der Corput-Halton sequences and their subsequences', Int. J. Number Theory 5 (2009), 719-746.

[8] R. Hofer and G. Larcher, 'Metric results on the discrepancy of Halton-Kronecker sequences', Math. Z. (2011), to appear. 
[9] R. Hofer and G. Larcher, 'On existence and discrepancy of certain Niederreiter-Halton sequences', Acta Arith. 141 (2010), 369-394.

[10] R. Hofer, F. Pillichshammer and G. Pirsic, 'Distribution properties of sequences generated by $q$-additive functions with respect to Cantor representation of integers', Acta Arith. 138 (2009), 179-200.

[11] L. Kuipers and H. Niederreiter, Uniform Distribution of Sequences (Wiley, New York, 1974).

[12] G. Larcher and H. Niederreiter, 'Generalized $(t, s)$-sequences, Kronecker-type sequences, and diophantine approximations of formal Laurent series', Trans. Amer. Math. Soc. 347 (1995), 2051-2073.

[13] C. Lemieux, Monte Carlo and Quasi-Monte Carlo Sampling, Springer Series in Statistics (Springer, New York, 2009).

[14] H. Niederreiter, 'Methods for estimating discrepancy', in: Applications of Number Theory to Numerical Analysis (ed. S. K. Zaremba) (Academic Press, New York, 1972), pp. 203-236.

[15] H. Niederreiter, 'Point sets and sequences with small discrepancy', Monatsh. Math. 104 (1987), 273-337.

[16] H. Niederreiter, Random Number Generation and Quasi-Monte Carlo Methods, CBMS-NSF Series in Applied Mathematics, 63 (SIAM, Philadelphia, 1992).

[17] H. Niederreiter, 'On the discrepancy of some hybrid sequences', Acta Arith. 138 (2009), 373-398.

[18] H. Niederreiter, 'Further discrepancy bounds and an Erdős-Turán-Koksma inequality for hybrid sequences', Monatsh. Math. 161 (2010), 193-222.

[19] F. Pillichshammer, 'Uniform distribution of sequences connected with the weighted sum-of-digits function', Unif. Distrib. Theory 2 (2007), 1-10.

[20] J. Spanier, 'Quasi-Monte Carlo methods for particle transport problems', in: Monte Carlo and Quasi-Monte Carlo Methods in Scientific Computing, Lecture Notes in Statistics, 106 (eds. H. Niederreiter and P. J.-S. Shiue) (Springer, New York, 1995), pp. 121-408.

\section{ROSWITHA HOFER, Institut für Finanzmathematik, Universität Linz, Altenbergerstr. 69, 4040 Linz, Austria \\ e-mail: roswitha.hofer@jku.at}

PETER KRITZER, Institut für Finanzmathematik, Universität Linz, Altenbergerstr. 69, $4040 \mathrm{Linz}$, Austria

e-mail: peter.kritzer@jku.at 